\title{
ON UNIMODALITY FOR LINEAR EXTENSIONS OF PARTIAL ORDERS*
}

\author{
F. R. K. CHUNG $\dagger$, P. C. FISHBURN $\dagger$ AND R. L. GRAHAM $\dagger$
}

\begin{abstract}
R. Rivest has recently proposed the following intriguing conjecture: Let $x^{*}$ denote an arbitrary fixed element in an $n$-element partially ordered set $P$, and for each $k$ in $\{1,2, \cdots, n\}$ let $N_{k}$ be the number of order-preserving maps from $P$ onto $\{1,2, \cdots, n\}$ that map $x^{*}$ into $k$. Then the sequence $N_{1}, \cdots, N_{n}$ is unimodal. This note proves the conjecture for the special case in which $P$ can be covered by two linear orders. It also generalizes this result for $P$ that have disjoint components, one of which can be covered by two linear orders.
\end{abstract}

1. Introduction. Given a finite partially ordered set $(P,<)$, where $<$ is asymmetric, we say that an injection $\lambda$ from $P$ into the set $Z$ of integers is a linear extension of $P$ if, for all $x, y \in P$,

$$
x<y \Rightarrow \lambda(x)<\lambda(y) .
$$

We shall presume that $P$ has $n$ elements and, in the main part of the paper, restrict ourselves to bijections $\lambda: P \rightarrow[n] \equiv\{1,2, \cdots, n\}$. Generalizations are discussed later.

Let $x^{*}$ be an arbitrary fixed element in $P$. For each $k \in[n]$, define $N_{k}$ to be the number of linear extensions $\lambda: P \rightarrow[n]$ for which $\lambda\left(x^{*}\right)=k$. Rivest [2] has proposed the following tantalizing conjecture.

CONJECTURE. The sequence $N_{k}, k \in[n]$, is unimodal.

By unimodal we mean that, for all $1 \leqq i<j<k \leqq n$,

$$
N_{j} \geqq \min \left\{N_{i}, N_{k}\right\} .
$$

In this note we shall prove that the conjecture is valid for the important class of partially ordered sets that can be partitioned into two linearly ordered subsets, i.e., chains, with <-pairs allowed between the chains. In fact, we show that the $N_{k}$ 's in this case satisfy the stronger property of logarithmic concavity, i.e.,

$$
N_{k}^{2} \geqq N_{k-1} N_{k+1} \quad \text { for } 1<k<n .
$$

A similar proof provides an interesting result involving the unimodality of certain sequences of integers.

2. Lattice paths in $\boldsymbol{Z}^{2}$. We shall say that the partially ordered set $(P, \prec)$ can be covered by two chains if there is a partition $\{A, B\}$ of $P$ such that the restriction of $<$ on each of $A$ and $B$ is a linear order. To avoid the trivial case, we shall suppose that $<$ on $P$ is not linear, and that $(P,<)$ can be covered by two chains, denoted as $A=$ $\left\{a_{1}<\cdots<a_{r}\right\}$ and $B=\left\{b_{1}<\cdots<b_{s}\right\}$, with $r \geqq 1, s \geqq 1$ and $r+s=n$. There can be "cross-relations" like $a_{i}<b_{j}$ or $b_{j}<a_{i}$ from $(P,<)$, but in any event $<$ must be asymmetric $(x<y \Rightarrow$ not $(y<x))$ and transitive.

Let $L$ denote the set of all ordered pairs of nonnegative integers. Each linear extension $\lambda: P \rightarrow[n]$ induces maps of $A$ and $B$ into $[n]$, with $\lambda\left(a_{1}\right)<\cdots<\lambda\left(a_{r}\right)$ and $\lambda\left(b_{1}\right)<\cdots<\lambda\left(b_{s}\right)$. To each such $\lambda$ we will associate a lattice path $\pi(\lambda)$ in $L$ as follows. The first point on $\pi(\lambda)$ is $(0,0)$. If the $k$ th point on $\pi(\lambda)$ is $\left(x_{k}, y_{k}\right)$ and if $\lambda(p)=k+1$, then the $(k+1)$ st point on $\pi(\lambda)$ is $\left(x_{k}+1, y_{k}\right)$ if $p \in A$, and $\left(x_{k}, y_{k}+1\right)$ if $p \in B$. The terminal point on $\pi(\lambda)$ is $(r, s)$. An example appears in Fig. 1.

\footnotetext{
* Received by the editors March 19, 1980.

$\dagger$ Bell Telephone Laboratories, Murray Hill, New Jersey 07974.
} 


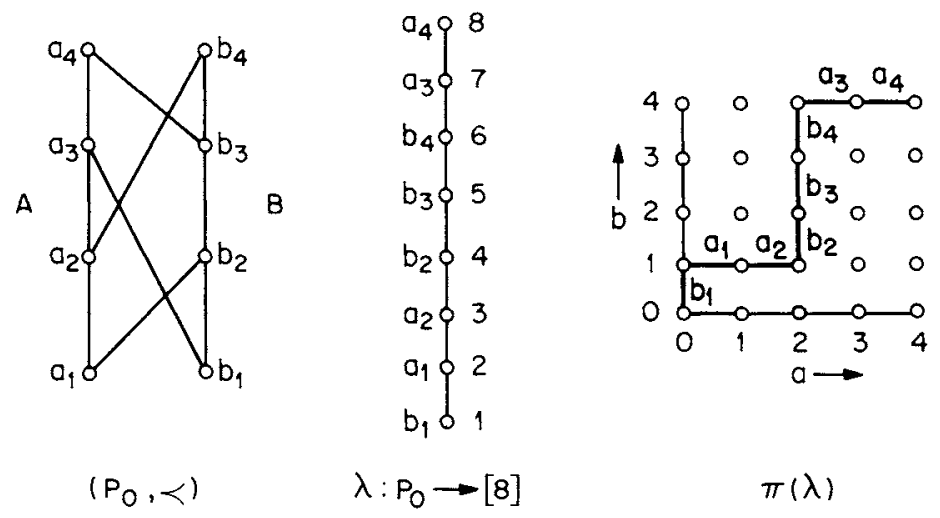

FIG. 1. The correspondence between $\lambda$ and $\pi(\lambda)$.

The fact that $\lambda$ preserves the linear orders on $A$ and $B$ is reflected in the fact that the indices of the $a_{i}$ and $b_{j}$ increase as we move along $\pi(\lambda)$ from $(0,0)$ to $(r, s)$. But how do the other <-pairs show up in $\pi(\lambda)$ ? For Fig. 1, what constraint does $a_{1}<b_{2}$ (which forces $\lambda\left(a_{1}\right)<\lambda\left(b_{2}\right)$ ) place on $\pi(\lambda)$ ? The answer is very simple. Each $a_{i}<b_{j}$ corresponds to a rectangular "barrier" which the path $\pi(\lambda)$ is not allowed to penetrate. This barrier is defined to be all lattice points $(x, y)$ in $L$ for which $x \leqq i$ and $y \geqq j-1$, as illustrated in Fig. 2.

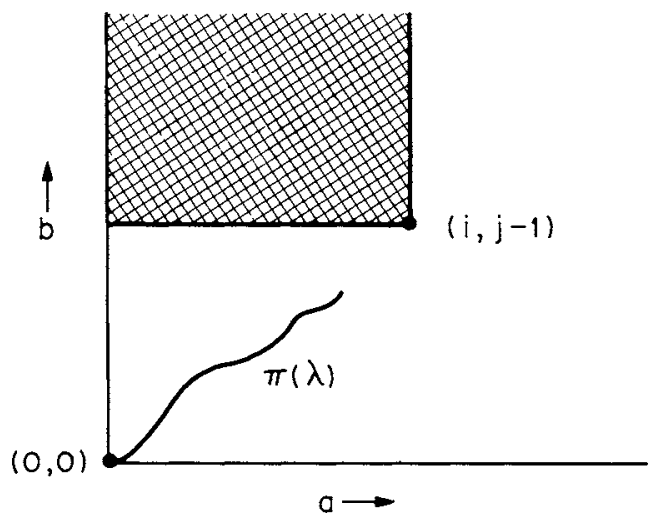

FIG. 2. The barrier for $a_{i}<b_{i}$.

The barrier for $a_{i}<b_{i}$ forces $\pi(\lambda)$ to reach a lattice point with $x$-coordinate $i$ before it reaches one with $y$-coordinate $j$, i.e., $a_{i}$ occurs before $b_{j}$ on $\pi(\lambda)$. This is precisely what is needed for $\lambda\left(a_{i}\right)<\lambda\left(b_{j}\right)$.

In a similar manner, $b_{j}<a_{i}$ corresponds to a rectangular barrier consisting of all $(x, y)$ in $L$ for which $x \geqq i-1$ and $y \leqq j$. For $\lambda$ to be a linear extension of $P, \pi(\lambda)$ must not penetrate any of the barriers formed from the cross-relations in $(P,<)$. Fig. 3 shows the union of the barriers for $\left(P_{0},<\right)$ from Fig. 1.

The next point we consider is how $\lambda\left(x^{*}\right)=k$ is reflected in $\pi(\lambda)$. Without loss of generality, we assume that $x^{*}=a_{i}$, so that $x^{*} \in A$. Then it is easy to see that $\lambda\left(a_{i}\right)=k$ iff $\pi(\lambda)$ contains the two points $(i-1, k-i)$ and $(i, k-i)$. (Similarly, $\lambda\left(b_{i}\right)=k$ iff $\pi(\lambda)$ contains $(k-j, j-1)$ and $(k-j, j)$.)

Suppose $N_{k-1}$ and $N_{k+1}$ are both positive, and let $\lambda^{+}$and $\lambda^{-}$be linear extensions of $P$ such that $\lambda^{+}\left(a_{i}\right)=k+1$ and $\lambda^{-}\left(a_{i}\right)=k-1$. Thus, $\pi\left(\lambda^{+}\right)$contains points $(i-1, k+1-$ 


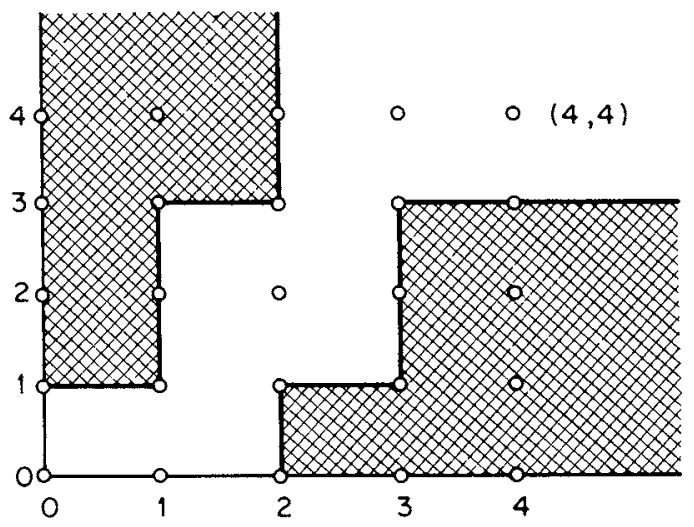

Fra. 3. The union of barriers for $\left(P_{0},<\right)$.

$i)$ and $(i, k+1-i)$, and $\pi\left(\lambda^{-}\right)$contains $(i-1, k-1-i)$ and $(i, k-1-i)$. Let $x_{0}$ be the largest integer that is $\leqq i-1$ such that, for some $y,\left(x_{0}, y+1\right)$ is on $\pi\left(\lambda^{+}\right)$and $\left(x_{0}, y\right)$ is on $\pi\left(\lambda^{-}\right)$, and let $y_{0}$, which cannot exceed $k-1-i$, be the largest integer such that $\left(x_{0}, y_{0}+1\right)$ is on $\pi\left(\lambda^{+}\right)$and $\left(x_{0}, y_{0}\right)$ is on $\pi\left(\lambda^{-}\right)$. Similarly, let $x_{1}$ be the smallest integer $\geqq i$ such that, for some $y,\left(x_{1}, y+1\right)$ is on $\pi\left(\lambda^{+}\right)$and $\left(x_{1}, y\right)$ is on $\pi\left(\lambda^{-}\right)$, and let $y_{1}$, which cannot be less than $k-i$, be the smallest integer such that $\left(x_{1}, y_{1}+1\right)$ is on $\pi\left(\lambda^{+}\right)$and $\left(x_{1}, y_{1}\right)$ is on $\pi\left(\lambda^{-}\right)$.

We now form two new lattice paths $\pi\left(\lambda_{1}\right)$ and $\pi\left(\lambda_{2}\right)$ as follows. Let $\pi\left(\lambda_{1}\right)$ consist of the points on $\pi\left(\lambda^{-}\right)$from $(0,0)$ to $\left(x_{0}, y_{0}\right)$, plus the points on $\pi\left(\lambda^{+}\right)$from $\left(x_{0}, y_{0}+1\right)$ to $\left(x_{1}, y_{1}+1\right)$ translated by -1 in the $y$-direction, plus the points on $\pi\left(\lambda^{-}\right)$from $\left(x_{1}, y_{1}\right)$ to $(r, s)$. Let $\pi\left(\lambda_{2}\right)$ consist of the points on $\pi\left(\lambda^{+}\right)$from $(0,0)$ to $\left(x_{0}, y_{0}+1\right)$, plus the points on $\pi\left(\lambda^{-}\right)$from $\left(x_{0}, y_{0}\right)$ to $\left(x_{1}, y_{1}\right)$ translated by +1 in the $y$-direction, plus the points on $\pi\left(\lambda^{+}\right)$from $\left(x_{1}, y_{1}+1\right)$ to $(r, s)$. It is of course possible to have $\pi\left(\lambda_{1}\right)=\pi\left(\lambda_{2}\right)$, or, equivalently, $\lambda_{1}=\lambda_{2}$, but this will not affect our conclusions. We observe that:

(i) $\pi\left(\lambda_{1}\right)$ and $\pi\left(\lambda_{2}\right)$ are lattice paths from $(0,0)$ to $(r, s)$ which contain $(i, k-i)$ and $(i-1, k-i)$, and, therefore, $\lambda_{1}\left(a_{i}\right)=\lambda_{2}\left(a_{i}\right)=k$;

(ii) since $\pi\left(\lambda^{+}\right)$lies strictly above $\pi\left(\lambda^{-}\right)$in the region where the translations occur in the construction, neither $\pi\left(\lambda_{1}\right)$ nor $\pi\left(\lambda_{2}\right)$ penetrates any of the barriers formed by $(P, \prec)$. It follows that $\lambda_{1}$ and $\lambda_{2}$ are linear extensions of $P$;

(iii) if two ordered pairs of the form $\left(\lambda^{+}, \lambda^{-}\right)$are distinct, then their associated $\left(\lambda_{1}, \lambda_{2}\right)$ pairs are distinct. This follows from the construction: if two $\left(\pi\left(\lambda^{+}\right), \pi\left(\lambda^{-}\right)\right)$ differ prior to $i$ on the abscissa, then their associated $\left(\pi\left(\lambda_{1}\right), \pi\left(\lambda_{2}\right)\right)$ will differ before $i$; if two $\left(\pi\left(\lambda^{+}\right), \pi\left(\lambda^{-}\right)\right)$differ after $i-1$, then their associated $\left(\pi\left(\lambda_{1}\right), \pi\left(\lambda_{2}\right)\right)$ will differ after $i-1$.

Thus, our construction provides an injection from the ordered pairs $\left(\lambda^{+}, \lambda^{-}\right)$into pairs $\left(\lambda_{1}, \lambda_{2}\right)$, where $\lambda^{+}$and $\lambda^{-}$are any linear extensions of $P$ for which $\lambda^{+}\left(a_{i}\right)=k+1$ and $\lambda^{-}\left(a_{i}\right)=k-1$, and $\lambda_{1}$ and $\lambda_{2}$ are linear extensions of $P$ that satisfy $\lambda_{1}\left(a_{i}\right)=\lambda_{2}\left(a_{i}\right)=$ $k$. If $\alpha, \beta$ and $\gamma$ are the number of linear extensions of $P$ for which $\lambda\left(a_{i}\right)=k+1$, $\lambda\left(a_{i}\right)=k-1$, and $\lambda\left(a_{i}\right)=k$, respectively, then such an injection requires $\gamma^{2} \geqq \alpha \beta$, for otherwise two $\left(\lambda_{1}, \lambda_{2}\right)$ pairs associated with distinct $\left(\lambda^{+}, \lambda^{-}\right)$pairs would have to be identical.

The preceding argument applies analogously when $x^{*}=b_{j}$. Thus, we have proved the following result.

THEOREM 1. Let $x^{*}$ be a fixed element in a partially ordered set $(P,<)$ on $n$ elements, and suppose $(P,<)$ can be covered by two chains. For $k \in\{1,2, \cdots, n\}$, let $N_{k}$ be the 
number of linear extensions $\lambda: P \rightarrow\{1,2, \cdots, n\}$ for which $\lambda\left(x^{*}\right)=k$. Then

$$
N_{k}^{2} \geqq N_{k-1} N_{k+1} \quad \text { for } k=2, \cdots, n-1 .
$$

COROLlaRY. Given the hypotheses of Theorem 1 , the sequence $N_{1}, N_{2}, \cdots, N_{n}$ is unimodal.

The same basic argument for Theorem 1 can be used to prove the following result for sequences of integers. Let $A=\left(a_{1} \geqq a_{2} \geqq \cdots\right)$ be a nonincreasing sequence of nonnegative integers. Given $A$, let $S_{n}$ be the number of nonincreasing sequences $x=\left(x_{1} \geqq x_{2} \geqq \cdots \geqq x_{n}\right)$ of integers for which $0 \leqq x_{k} \leqq a_{k}$, for $k=1, \cdots, n$.

THEOREM 2. The sequence $S_{1}, S_{2}, \cdots$ is logarithmically concave, i.e.,

$$
S_{n}^{2} \geqq S_{n-1} S_{n+1} \quad \text { for all } n \geqq 2 \text {. }
$$

When $A$ is constant, say $A=(t, t, t, \cdots)$, Theorem 2 shows the (easily proved) logarithmic concavity of the binomial coefficients $\left(\begin{array}{c}t+k \\ k\end{array}\right)$ for $k=1,2, \cdots$.

3. A generalization. We now generalize our analysis of logarithmic concavity by considering disjoint partial orders along with linear extensions that map $P$ into $[m] \equiv\{1, \cdots, m\}$ when $m$ exceeds the cardinality of $P$. The following lemma provides a basis for the generalization.

LEMMA. Let $(P,<)$ and $(P \cup C,<)$ be partially ordered sets on $n$ and $n+\alpha$ elements, respectively, that have the same ordered pairs in their partial orders with $C \cap P=\varnothing$. Let $x^{*} \in P$ be fixed, and let $N_{k}$ and $N_{k}^{\prime}$, respectively, be the number of linear extensions $\lambda: P \rightarrow[n]$ and $\lambda^{\prime}: P \cup C \rightarrow[n+\alpha]$ that have $\lambda\left(x^{*}\right)=k$ and $\lambda^{\prime}\left(x^{*}\right)=k$. If $N_{1}, \ldots, N_{n}$ is logarithmically concave, then so is $N_{1}^{\prime}, \cdots, N_{n+\alpha}^{\prime}$.

If $C$ is empty, there is nothing to prove; so suppose initially that $C=\{c\}$, with $\alpha=1$. Since neither $c<x$ nor $x<c$ for each $x \in P$, each $\lambda$ for $P$ generates $n+1 \lambda^{\prime}$ for $P \cup\{c\}$ according to the $n+1$ placements of $c$. With $N_{0}=N_{n+1}=0$,

$$
N_{k}^{\prime}=(k-1) N_{k-1}+(n-k+1) N_{k} \quad \text { for } k=1, \cdots, n+1 .
$$

Using this relationship, $\left(N_{k}^{\prime}\right)^{2}-N_{k-1}^{\prime} N_{k+1}^{\prime}$, for $2 \leqq k \leqq n$, reduces to

$$
\begin{gathered}
k(k-2)\left[N_{k-1}^{2}-N_{k-2} N_{k}\right]+(n-k)(n-k+2)\left[N_{k}^{2}-N_{k-1} N_{k+1}\right] \\
+(k-2)(n-k)\left[N_{k-1} N_{k}-N_{k-2} N_{k+1}\right]+\left(N_{k-1}-N_{k}\right)^{2},
\end{gathered}
$$

which must be nonnegative if $\left\{N_{k}\right\}$ is logarithmically concave.

This completes the proof of the lemma if $\alpha \leqq 1$, so suppose in this paragraph that $\alpha \geqq 2$ with $C=\left\{c_{1}, \cdots, c_{\alpha}\right\}$. The $\lambda^{\prime}: P \cup C \rightarrow[n+\alpha]$ can be generated from the $\lambda: P \rightarrow$ [n] by adding one $c_{i}$ at a time. For a given $\lambda$, we first add $c_{1}$ to obtain $n+1$ linear extensions from $P \cup\left\{c_{1}\right\}$ onto $[n+1]$; for each of these $n+1$, we then add $c_{2}$ to obtain $n+2$ linear extensions from $P \cup\left\{c_{1}, c_{2}\right\}$ onto $[n+2]$; and so forth. If $\left\{N_{m}\right\}$ is logarithmically concave, then successive applications of the result obtained in the preceding paragraph for each $c_{i}$ addition show that $\left\{N_{k}^{\prime}\right\}$ must be logarithmically concave. The lemma is thus proved.

We now state our generalization, discuss its features, and then conclude this section with its proof.

THEOREM 3. Suppose $\left(P_{1},<_{1}\right),\left(P_{2},<_{2}\right)$ and $(P,<)$ are partially ordered sets on $n_{1}$, $n_{2}$ and $n$ elements respectively such that $0<n_{1} \leqq n, P_{1} \cup P_{2}=P, P_{1} \cap P_{2}=\varnothing$ and $<_{1} \cup<_{2}=<$. Let $x^{*} \in P_{1}$ be fixed, and let $N_{k}\left(k=1, \cdots, n_{1}\right)$ be the number of linear extensions $\lambda: P_{1} \rightarrow\left[n_{1}\right]$ for which $\lambda\left(x^{*}\right)=k$. In addition, given $m \geqq n$, let $M_{k}(k=$ 
$1, \cdots, m)$ be the number of linear extensions $\lambda^{*}: P \rightarrow[m]$ for which $\lambda^{*}\left(x^{*}\right)=k$. If $N_{1}, \cdots, N_{n_{1}}$ is logarithmically concave, then so is $M_{1}, \cdots, M_{m}$.

When $n_{2}=0$ and $m>n$, this shows that logarithmic concavity for $\lambda: P \rightarrow\lfloor n\rfloor$ carries over to $\lambda^{*}: P \rightarrow[m]$. When $n_{2}>0$ and $m=n$, Theorem 3 says that logarithmic concavity for the elements within a part of $(P,<)$, namely $\left(P_{1},<_{1}\right)$, carries over to all of $(P,<)$ for those same elements, provided that the rest of $(P,<)$ is not connected to the first part. The combination of these two cases provides the generalization stated in the theorem.

Theorems 1 and 3 together yield the following result.

THEOREM 4. If an n-element partially ordered set $(P,<)$ can be partitioned into partially ordered sets $\left(P_{1},<_{1}\right)$ and $\left(P_{2},<_{2}\right)$ with no $<$-connection between $P_{1}$ and $P_{2}$, if $\left(P_{1},<_{1}\right)$ can be covered by two chains, and if $x^{*} \in P_{1}, m \geqq n$, and $M_{k}$ is the number of linear extensions $\lambda: P \rightarrow[m]$ for which $\lambda\left(x^{*}\right)=k$, then $M_{1}, \cdots, M_{m}$ is logarithmically concave and unimodal.

We now sketch the proof of Theorem 3 using the notation in its statement. In addition, let $T_{k}$ be the number of linear extensions $\lambda_{0}: P \rightarrow[n]$ for which $\lambda_{0}\left(x^{*}\right)=k$, and if $n_{2}>0$, let $\beta$ be the number of linear extensions $\lambda_{2}: P_{2} \rightarrow\left[n_{2}\right]$, and let $N_{k}^{\prime}$ be the number of linear extensions $\lambda^{\prime}: P_{1} \cup C \rightarrow[n]$ that have $\lambda^{\prime}\left(x^{*}\right)=k$ when $C$ is a completely unordered $n_{2}$-element set (see the lemma) that is disjoint from $P_{1}$.

If $n_{2}=0$ then $T_{k}=N_{k}$, so assume henceforth in this paragraph that $n_{2}>0$. We shall apply the lemma with $\alpha=n_{2}$. Consider a fixed $\lambda_{2}: P_{2} \rightarrow\left[n_{2}\right]$ along with a generic $\lambda_{1}: P_{1} \rightarrow[n]$. The $n_{2}$ numbers in $[n]$ that are not in $\lambda_{1}\left(P_{1}\right)$ can be bijectively assigned to the elements in $P_{2}$ in exactly one way that preserves the $\lambda_{2}$ order and yields a $\lambda_{0}: P \rightarrow[n]$-as compared to the $n_{2}$ ! ways this could be done for the unordered set $C$. Since this is true for each such $\lambda_{1}$, it follows that the number of $\lambda_{0}: P \rightarrow[n]$ that have $\lambda_{0}\left(x^{*}\right)=k$ and have $P_{2}$ in its $\lambda_{2}$ order is $N_{k}^{\prime} / n_{2}$ !. Since there are $\beta$ such $\lambda_{2}, T_{k}=$ $\beta N_{k}^{\prime} / N_{2}$ !. If $N_{1}, \cdots, N_{n_{1}}$ is logarithmically concave, then the lemma says that $T_{1}, \cdots, T_{n}$ is too.

This proves Theorem 3 if $m=n$. If $m>n$, we reapply the lemma with $\alpha=m-n$. In this case let $C^{\prime}$ be a completely unordered $(m-n)$-element set disjoint from $P$ and, with respect to $\left(P \cup C^{\prime},<\right)$, let $T_{k}^{\prime}$ be the number of linear extensions $\lambda^{\prime}: P \cup C^{\prime} \rightarrow[m]$ for which $\lambda^{\prime}\left(x^{*}\right)=k$. By the lemma, if $\left\{T_{k}\right\}$ is logarithmically concave then so is $\left\{T_{k}^{\prime}\right\}$. Since the $m-n$ numbers in $[m]$ that aren't in a $\lambda^{\prime}(P)$ can be bijectively assigned to $C^{\prime}$ in $(m-n)$ ! ways, it follows that $M_{k}$ as defined in Theorem 3 equals $T_{k}^{\prime} /(m-n) !$. When this is combined with preceding conclusions, we see that if $N_{1}, \cdots, N_{n_{1}}$ is logarithmically concave, then so is $M_{1}, \cdots, M_{m}$.

4. Concluding remarks. The preceding techniques can be used to prove other unimodality results for restricted lattice path problems. For example, consider lattice paths $\pi$ that are not allowed to penetrate barriers of the type shown in Fig. 3, so that $\pi$ is bounded between two increasing staircases. Let $D_{n, k}$ be the number of such paths that go through point $(k, n-k)$. Then, for each $n$, the sequence $D_{n, k}, 0 \leqq k \leqq n$, is logarithmically concave and therefore unimodal. (Of course, here we are just looking at the intersections of lattice paths with the line $x+y=n$.) The reader is referred to the recent paper of Graham, Yao, and Yao [1] for similar applications of these ideas.

Finally, we note another open conjecture that is suggested by our analysis. Within the context used for the earlier conjecture, we propose:

CONJECTURE*. The sequence $N_{k}, k \in[n]$, is logarithmically concave.

Conjecture* is stronger than Rivest's Conjecture since unimodality follows from logarithmic concavity, but not conversely. Thus, a counterexample for Conjecture* need not disprove unimodality, while verification of Conjecture* would establish Rivest's Conjecture. 
Note added in proof. R. Stanley has just proved Conjecture* using a very ingenious application of the Alexandroff-Fenchel theorem (which guarantees the logarithmic concavity of certain coefficients arising from the volume of weighted sums of $n$ dimensional polytopes).

\section{REFERENCES}

[1] R. L. GRAhAM, A. C. YAO, AND F. F. YAO, Some monotonicity properties of partial orders, this Journal, 1 (1980), pp. 251-258.

[2] R. RIVES' (personal communication). 